\title{
Modular Design of Hydromechanical Transmissions for Mobile Working Machines
}

\author{
K. Pettersson and P. Krus \\ Division of Fluid and Mechatronic Systems, Department of Management and Engineering, Linköping University, Sweden \\ E-mail: karl.pettersson@liu.se, petter.krus@liu.se
}

\begin{abstract}
This paper demonstrates an optimisation-based method of designing modular gearboxes scalable for a range of applications. The design is adapted to the typical operating behaviours of the reference vehicles and considers the manufacturing costs of the gearboxes. Hydromechanical continuously variable transmissions (CVTs) are today strong candidates to replace drive line transmissions based on fuel-thirsty torque converters in many mobile working machines. The advantages include a wide range of torque/speed ratios, high energy efficiency throughout the speed range and decoupling of engine speed and vehicle speed. Advanced multiple mode CVTs, however, are difficult to evaluate early in the product development process due to their complex architectures and the great variety of possible concepts. There is consequently an increased need for methods to design, compare and evaluate the transmission concepts. To decrease the development and manufacturing costs, there is also a need for scalable transmission concepts that can be used in several applications of different classes. The results show the proficiency of the methodology compared to a manual design process and that the energy efficiency of the transmissions are strongly linked to the designs.
\end{abstract}

Keywords: Hydromechanical transmissions, Power-split, Design optimisation

\section{Introduction}

A multiple-mode hydromechanical transmission provides a wide torque/speed range with a high efficiency throughout the speed range and is today a strong candidate to replace the torque converter in many heavy mobile working machines. Furthermore, the continuously variable torque/speed ratio allows for decoupling of engine speed and vehicle speed which enables more energy savings through engine management. With single-mode configurations, such as pure hydrostatic transmissions, very large displacement machines (hydraulic pump/motors) are required to meet the high power demands of heavier applications. By using synchronised shafts, multiple hydromechanical configurations can be combined to improve the energy efficiency and decrease the necessary sizes of the displacement machines. In recent years, a number of commercial concepts have been released for construction machines and off-highway applications, see for instance [1].

The combination of configurations leads to great many possible multiple-mode concepts. Furthermore, complex multiple-mode transmissions contain large numbers of mechanical gears and are more difficult to design. The notion of designing the transmission means, in this paper, the process of sizing the transmission components, i.e. the gear ratios and the displacement machines. For complex transmission architectures, there is a design space in which the transmission has the same performance, i.e. two different designs can result in the same performance. A fair comparison between concepts is consequently only valid for optimised designs.

Much research has been done on developing simulation models for hydromechanical transmissions with the purpose of evaluating different transmission concepts, see for instance [2], [3] and [4]. Comparisons and evaluations of different transmission concepts can be found in for instance [5] and [6]. Fewer works, however, can be found on the design process of hydromechanical transmissions. Volpe et al. [7] suggested an optimisation-based design method using only kinematic models of the transmission components. Macor and Rosetti formulated a simulation-based optimisation method using more detailed loss models in [8] and [9]. The authors of this paper have proposed a design methodology for complex hydromechanical transmissions based on numerical optimisation in [10]. The methodology adapts the design in order to minimise the energy consumption for a predefined operating behaviour of the considered application. The studies have shown that the design of the transmission is strongly linked to the energy consumption of the application. It is also shown that an optimal design may be difficult to find manually, underlining the need for an automated design process. 


\subsection{Modular Design}

The concept of modularity and platform design within product development is a well-recognised methodology to decrease the variety of components in a product family. The main advantages include lower manufacturing costs and lower development costs due to the increased communality of the products. The trade-off for the company lies instead in the decreased individual performance of the products. [11]

\subsection{Problem Outline}

There are a number of plausible desired characteristics of a gearbox, e.g. energy efficiency, manufacturing cost, controllability, low volume and weight. For the industrial designer these are most likely all aspects to consider when designing the transmission. For an OEM with a range of vehicles in its product portfolio, more than one application needs to be considered in the development process. A scalable (modular) gearbox with collective characteristics can reduce both the development costs and the manufacturing costs of the transmissions. This paper focuses on the design process of a modular gearbox suitable for a range of applications. The modularity is here confined to the use of the same transmission concept with the same hydraulic variator, but with varying number of modes. The modular design targets a wide range of wheel loader applications of different power classes.

\section{Transmission Concept}

The concept considered in the design methodology is the so called "Jarchow-concept" with an arbitrary number of modes, shown in fig. 1.

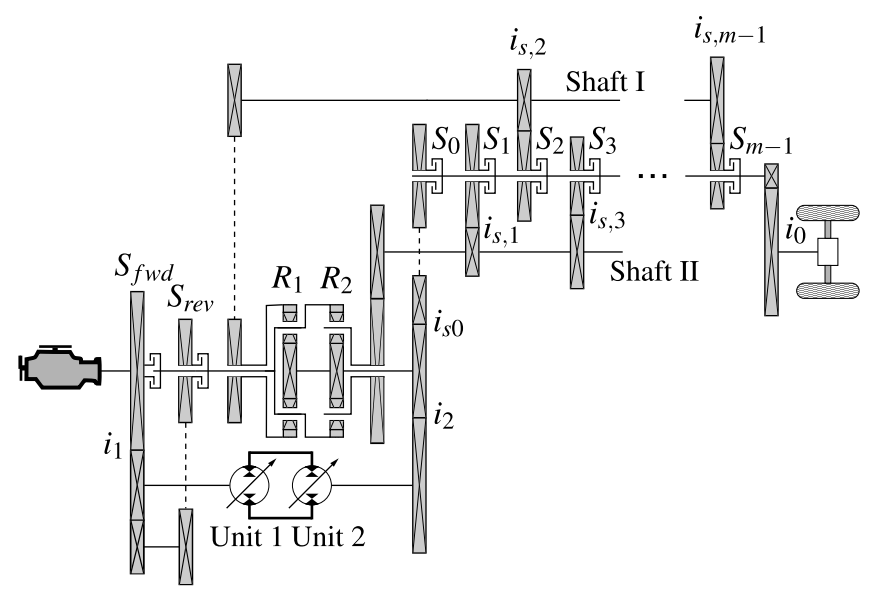

Figure 1: The "Jarchow-concept" with an arbitrary number of modes. The dimensions of the gear wheels are not necessarily proportionally correct; the figure merely shows the principle of the concept. In the figure, the number of modes, $m$, is an odd number.

The starting mode $(\mathrm{H})$ is purely hydrostatic and the subsequent modes are input-coupled power-split modes. The number of modes, $m$, is defined as the number of forward power-split-modes plus the hydrostatic mode, i.e. $m=3$ means one hydrostatic mode and two power-split modes for the forward motion. There are also $m-1$ power-split modes for the reverse motion. In the odd power-split modes (F1, F3 etc), the second planetary gear $\left(R_{2}\right)$ actively transfers power to Shaft II and in the even power-split modes (F2, F4 etc) the first planetary gear $\left(R_{1}\right)$ actively transfers power to Shaft I. The complete clutch configuration is shown in Table 1. An ar-

Table 1: Clutch arrangement

\begin{tabular}{|c|c|c|c|c|c|}
\hline Mode & $S_{f w d} S_{r e v}$ & $S_{0}$ & $S_{1}$ & $S_{2}$ & $S_{m-1}$ \\
\hline Rm-1 & $\bullet$ & & & & $\bullet$ \\
\hline R2 & $\bullet$ & & & $\bullet$ & \\
\hline R1 & $\bullet$ & & $\bullet$ & & \\
\hline $\mathrm{H}$ & & $\bullet$ & $\bullet$ & & \\
\hline F1 & $\bullet$ & & $\bullet$ & & \\
\hline F2 & $\bullet$ & & & $\bullet$ & \\
\hline Fm-1 & $\bullet$ & & & & $\bullet$ \\
\hline
\end{tabular}

bitrary number of modes makes it possible to scale the transmission up or down depending on the power class of the application. The principle of this architecture can be found in several commercial hydromechanical CVTs and it has been treated previously in some of the papers referenced above.

\section{Reference Vehicles}

This study focus on the design of a modular gearbox for three wheel loader applications to demonstrate the design methodology. All three wheel loader sizes are traditionally equipped with a torque converter and a powershift gearbox making them suitable applications for hydromechanical transmissions. Table 2 shows the main parameters of the considered wheel loaders, denoted A-C, where A is the largest and $\mathrm{C}$ is the smallest. Figure 2 shows the tractive force requirements.

Table 2: Wheel loader parameters.

\begin{tabular}{l|cccl}
\hline Wheel loader & $\mathbf{A}$ & $\mathbf{B}$ & $\mathbf{C}$ & Unit \\
\hline$P_{\max }$ & 219 & 161 & 96 & $\mathrm{~kW}$ \\
\hline$F_{\max }$ & 245 & 181 & 130 & $\mathrm{kN}$ \\
\hline$v_{\max }$ & 40 & 40 & 40 & $\mathrm{~km} / \mathrm{h}$ \\
\hline
\end{tabular}

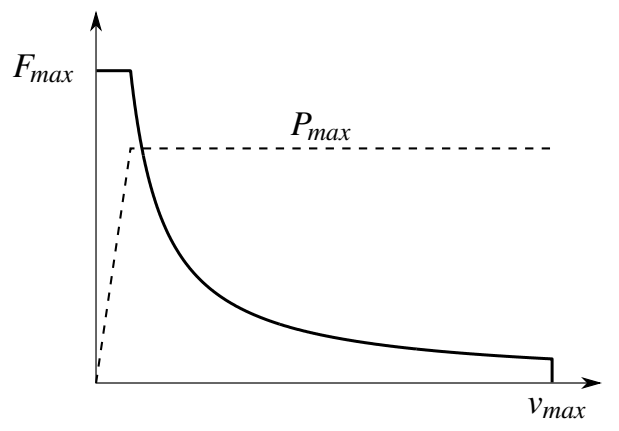

Figure 2: The tractive force requirements of a wheel loader. 
To evaluate the energy consumption of a transmission design, pre-recorded data from typical operating cycles are used. By simulating the operating cycles, the energy consumption of a transmission design can be evaluated. This is a common way of evaluating system concepts and allows for an optimised system design specifically for the typical operating behaviour of the vehicle [12]. Wheel loaders are versatile machines and have many different application areas. Many machines are sold as multi-purpose machines although operating with a bucket in a production site is the most common task [13]. Two operating cycles are often used to characterise the operating behaviour of the wheel loader; the short loading cycle and the load-carry cycle, see fig. 3. For details of the operation of these cycles see [14].

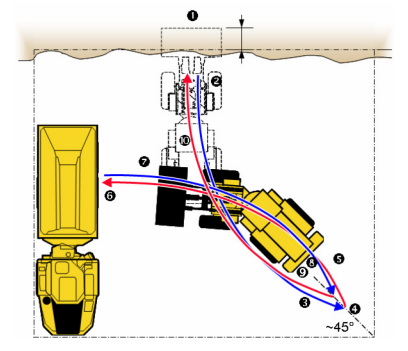

(a) Short loading cycle

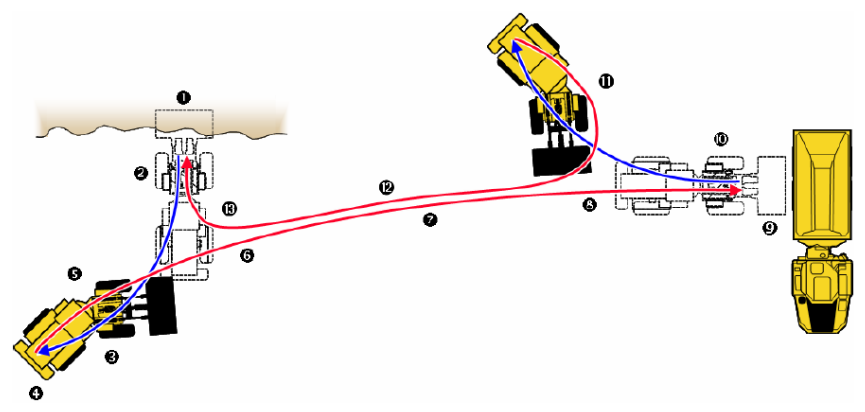

(b) Load-carry cycle

Figure 3: Typical operating cycles of the wheel loader, from [14].

Two examples of the cycles above have been recorded in real world operation for all three wheel loaders. For both cycles, gravel is loaded from a pile on to a load receiver of appropriate height for each wheel loader. Two trained operators each performed nine cycles each for every wheel loader application in every operating cycle. Data was recorded for the output axle of the transmission. The data was then analysed and one representative data set was selected for every wheel loader and cycle. This data is then used to represent the typical operating cycles for each wheel loader application.

Normally, larger wheel loaders are used to a greater extent in short loading cycles whereas smaller machines more often operate in transportation phases. The complete operating behaviour of the wheel loader is therefore achieved by weighing each cycle according to its significance for each wheel loader application. The weight factors are supplied by an OEM and represent approximate values of the distribution for the average wheel loader. Figure 4 shows the operating point distribution over the speed range for the complete operating behaviour of the wheel loaders. The suggested

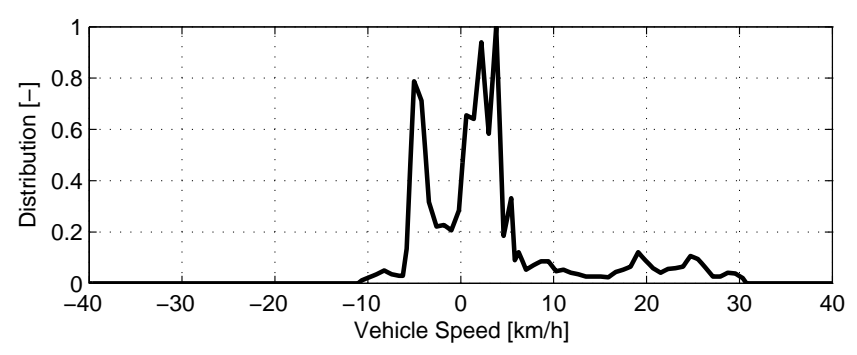

(a) Wheel loader A

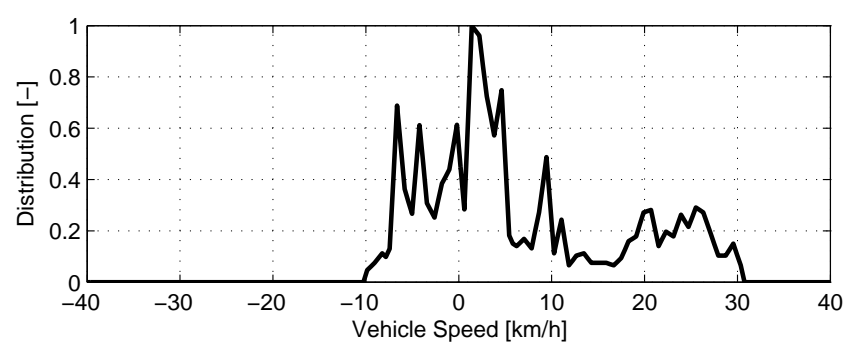

(b) Wheel loader B

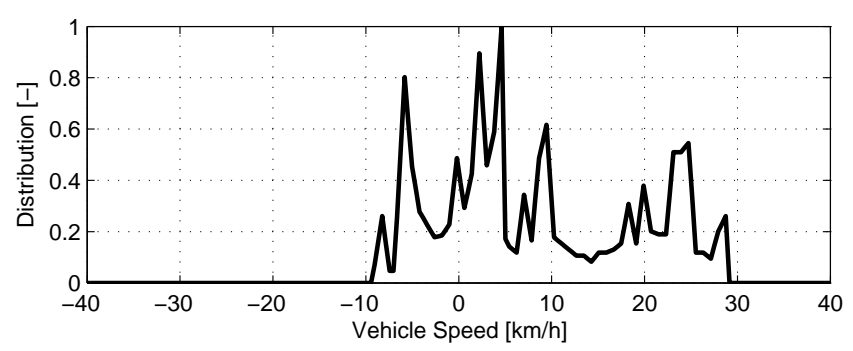

(c) Wheel loader C

Figure 4: Distribution of the operating speeds for the typical operating behaviour of the considered wheel loaders

operating cycles represent a typical behaviour of the machine and, as stated earlier, are not true for every (any) wheel loader in the field. The use of typical operating cycles, however, is still helpful in the design process [12].

\section{Transmission Design}

When dealing with multiple-mode hydromechanical transmissions, much can be gained from using similar concepts for many applications, including different power classes of the same type of vehicle. The development costs are significantly reduced if the gearbox can be scaled up and down or at least easily adapted to each application. Additionally, manufacturing costs can be reduced by producing or buying larger quantities of the same component. 


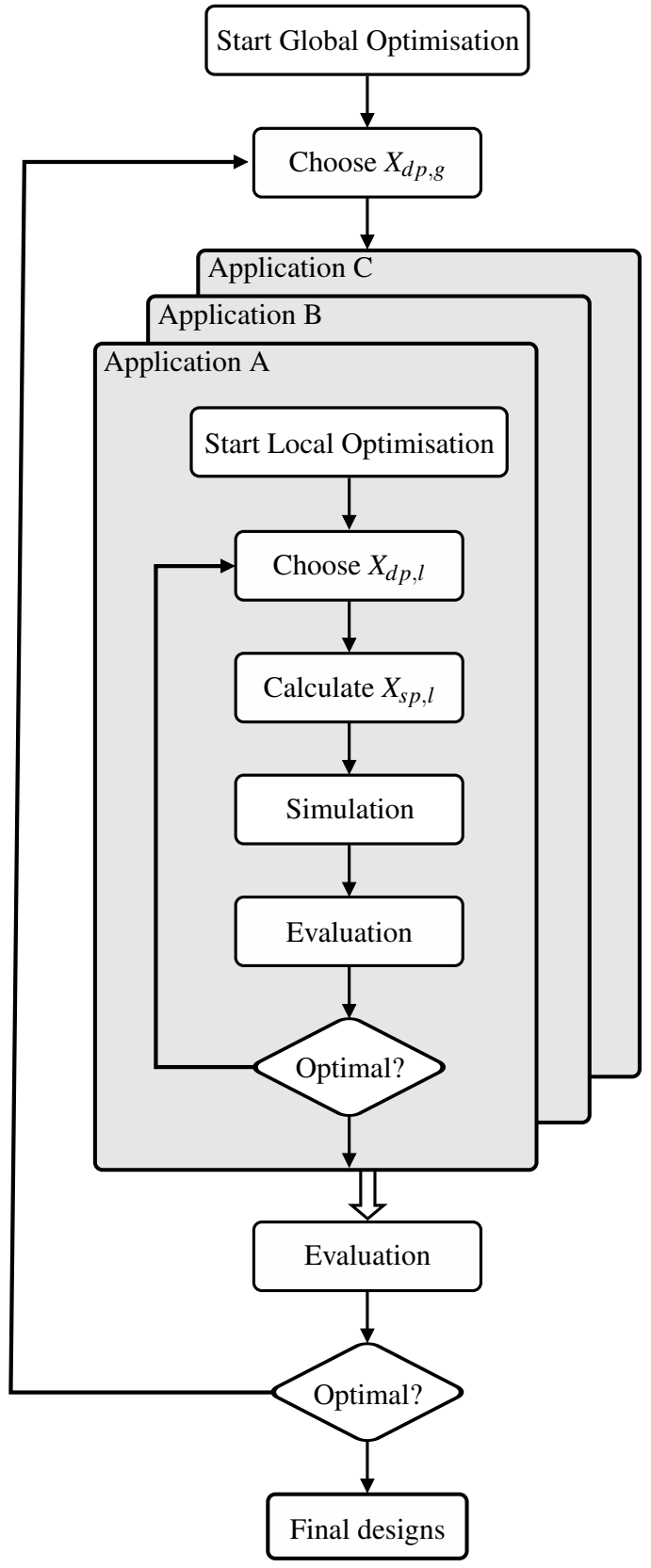

Figure 5: The design process for the modular gearbox. The inner loop optimises the transmission designs for all wheel loaders individually given a number of modes and a variator size. The outer loop sets the number of modes and variator size optimally for the considered range of applications.

The tractive force requirements and the demands of the gearbox characteristics naturally vary between applications. The demand for low cost and high energy efficiency may vary greatly also within different sizes of the same type of vehicle. One approach to achieve a modular gearbox is to use the same number of modes in each transmission and to vary the sizes of the displacement machines to match the tractive force requirements of each application, as shown in [1]. Another approach is to use the same hydrostatic variator, i.e. the same sizes of displacement machines, but with different numbers of modes depending on tractive power demands.
The advantages include lower manufacturing costs for the gearboxes since the variator can be bought or manufactured in larger series. The development costs may also be reduced and robustness increased if similar control software can be used for all transmissions. This is the design approach further examined in this paper. The trade-off for this scenario is that transmissions with a small hydraulic variator, leading to lower power losses, requires a higher number of modes, leading to higher costs.

The proposed design methodology is an extension of the previously developed methodology for designing complex hydromechanical transmissions [10]. Briefly explained, the design is achieved by identifying the degrees of freedom for the concept and letting an optimisation routine set the design parameters to minimise the energy consumption during simulated work cycles. The modular transmission is achieved when the design of each application is optimised with a collective hydraulic variator. The choice of hydraulic variator and number of modes for each application should consequently also be the subject of a design optimisation. Figure 5 shows the proposed methodology for the design process.

\subsection{Local Optimisation}

The local optimisation loop optimally designs each application one by one, with a given number of modes $m$ and a given variator size $D_{v a r}$. The explicit design relations have previously been derived in [10] for the same concept, but with Unit 2 as a fixed displacement machine. The two degrees of freedom are shown in eq. (1):

$$
\begin{aligned}
& x_{1}=\frac{v_{s h i f t, k+1}}{v_{s h i f t, k}} \text { for } \mathrm{k}=1,3,5, \ldots \\
& x_{2}=\frac{v_{s h i f t, k+1}}{v_{\text {shift }, k}} \text { for } \mathrm{k}=2,4,6, \ldots
\end{aligned}
$$

The degrees of freedom are hence the freedom of positioning the mode shifts within the speed range of the vehicle. With a variable Unit 2, smaller displacement machines may be required, since the hydrostatic speed range increases. For this study, the minimum displacement of Unit 2, $\varepsilon_{2, \min }$, is left as a design parameter, since it is unclear whether a lower $\varepsilon_{2, \min }$ will lead to higher energy efficiency. Generally, the efficiency of the displacement machine is reduced when operating with low relative displacements. The system parameters and the design parameters for the local optimisation are consequently:

$$
\begin{aligned}
& X_{s p, l}=\left[\begin{array}{lllllllllll}
D_{1} & D_{2} & R_{1} & R_{2} & i_{1} & i_{2} & i_{s, 0} & i_{s, 1} & i_{s, 2} & \ldots & i_{s, m-1}
\end{array}\right]^{T} \\
& X_{d p, l}=\left[\begin{array}{lll}
x_{1} & x_{2} & \varepsilon_{2, \min }
\end{array}\right]^{T}
\end{aligned}
$$

The simulation of the transmission is based on backward calculation with known steady-state models of the components. The greatest power losses come from the displacement machines, which require detailed loss models with dependency on all operating conditions [4]. The hydraulic variator also requires a charge pump for anti-cavitation and cooling and to supply the control circuit. For details of the simulation models used in this paper, see [10]. 


\subsubsection{Objective Function}

The objective function for the local optimisation is simply the simulated equivalent energy consumption of the transmission for the specified operating cycles, shown in eq. (3):

$$
f_{l}=\sum_{k} w_{l, k} \int_{0}^{t_{\text {end }, k}} P_{\text {tot }, k}(t) d t
$$

where $w_{k}$ is the weight factor for the operating cycle, as described in section 3 .

\subsubsection{Constraints}

Two constraints are used in the local optimisation to restrict the freedom of positioning the mode shifts in the speed range, see eq. (4).

$$
\begin{aligned}
v_{\text {shift }, 1} & \geq 5 \mathrm{~km} / \mathrm{h} \\
v_{\text {shift }, k+1}-v_{\text {shift }, k} & \geq 2 \mathrm{~km} / \mathrm{h} \text { for } \mathrm{k}=1,2, \ldots, \mathrm{m}-1
\end{aligned}
$$

The first constraint relates to the need to avoid mode shifts during the bucket fill operation, which normally takes place at under $5 \mathrm{~km} / \mathrm{h}$, see fig. 4 . The second constraint prevents the mode shifts to be positioned too closely together. Between two mode shifts, the relative displacement of Unit 1 is controlled from -1 to 1 or vice versa. During quick accelerations and decelerations, there are consequently high demands on the control speed of the displacement machines.

\subsection{Global Optimisation}

The global optimisation loop sets the optimal size of the variator and the number of modes for each wheel loader. The design parameters for the global optimisation are consequently:

$$
X_{d p, g}=\left[\begin{array}{ll}
m & D_{v a r}
\end{array}\right]^{T}
$$

where

$$
\begin{aligned}
m & =\left[\begin{array}{lll}
m_{A} & m_{B} & m_{C}
\end{array}\right]^{T} \\
D_{v a r} & =D_{1}+D_{2}
\end{aligned}
$$

\subsubsection{Objective Function}

The target for the global optimisation problem is a multiobjective one. Since energy efficiency normally increases with number of modes, it is unreasonable only to optimise the vehicles' energy efficiency. The objective should instead formulate the trade-off between energy efficiency and the manufacturing cost of the gearbox.

$$
f\left(X_{d p, g}\right)=\lambda_{1} f_{g 1}+\lambda_{2} f_{g 2}
$$

The first objective, $f_{g 1}$, is the weighted sum of equivalent energy consumptions from the local optimisation results according to eq. (8).

$$
f_{g 1}=\sum_{k} w_{g 1, k} E_{k, \text { equiv }} \text { for } k=A, B \text { and } C
$$

The weight factors $w_{g 1, k}$ specify the importance of energy efficiency for one application versus another. These values relate to factors such as number of sold vehicles, cost margins, fuel price, customer demands, acceptable payback period, etc. This type of analysis is beyond the scope of this paper; instead, the equivalent energy consumption is evenly weighted for the considered wheel loaders. The second objective, $f_{g 2}$, is the weighted manufacturing costs of the gearboxes, according to eq. (9).

$$
f_{g 2}=\sum_{k} w_{g 2, k} C_{k} \text { for } k=A, B \text { and } C
$$

The weight factors $w_{g 2, k}$ also relate to the above mentioned market-related factors and are here assumed to be equal. The manufacturing costs are simply modelled as the sum of all component costs plus a fixed cost for the housing, additional hydraulic components, etc. See [15].

\subsubsection{Constraints}

In previous studies, the sizes of the displacement machines are calculated and can take any continuous value. A more realistic approach for an industrial designer is to choose from a discrete series of sizes. To represent this scenario, the listed sizes of the Bosch Rexroth standard displacement machines A4VG/40 (Unit 1) and the A6VM/71 (Unit 2) [16] are used as possible variator sizes. One variator size means, for this case, only one combination of machine sizes, according to eq. (10).

$$
\left[\begin{array}{l}
D_{1} \\
D_{2}
\end{array}\right] \in\left(\left[\begin{array}{l}
145 \\
170
\end{array}\right] ;\left[\begin{array}{l}
175 \\
215
\end{array}\right] ;\left[\begin{array}{l}
210 \\
280
\end{array}\right]\right)
$$

The number of modes for every transmission is naturally also a discrete value, which in this study is limited according to eq. (11).

$$
2 \leq m_{k} \leq 6 \text { for } k=A, B \text { and } C
$$

\subsection{Optimisation Algorithm}

The Complex-RF method is used in the design process for both the global and the local optimisation. Briefly explained, the algorithm generates a certain number of random points in the design space and reflects the worst point towards the centroid of the other points until it is no longer the worst point. This process is iterated until the minimum function value is found or the maximum number of evaluations is reached. The Complex-RF method has previously been proved to be suitable to use in simulation-based design optimisation. [17]

\section{Results and Discussions}

Figure 6 shows optimisation results for consecutive runs with linearly changed weight factors $\lambda_{1}$ and $\lambda_{2}$ to form the Pareto optimal front. The solution will generally travel to the bottom right corner of the graph with an increased number of modes for the transmissions. A suitable weight between the two objectives is probably found somewhere in the middle, where a higher number of modes for a larger application is a reasonable choice. The awkward selections of $m$ on the Pareto front shows that an optimised manual design would be 


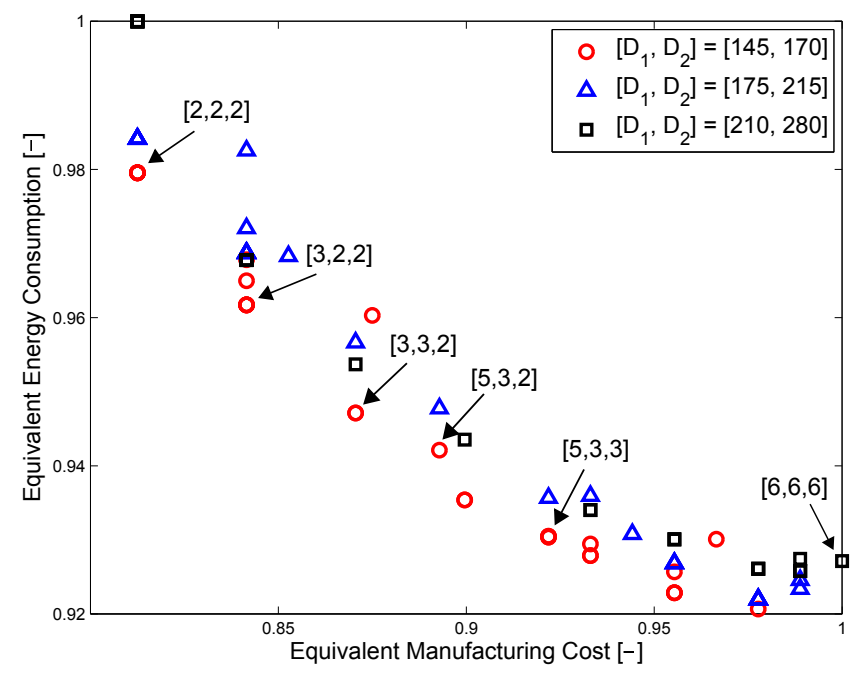

Figure 6: The Pareto front for the multi-objective design optimisation. Each marker represents a set of numbers of modes as indicated, according to $\left[m_{A}, m_{B}, m_{C}\right]$. The top left corner represents low energy efficiency and low costs, whereas the bottom right corner represents high costs and high energy efficiency.

difficult to achieve. The absence of the 4-mode transmission can also be seen in the graph. The 4-mode transmission seems to be only slightly better than the 3-mode transmission that dominates the Pareto front. This can also be seen in fig. 7 where the optimised designs of the transmissions for wheel loader $\mathrm{C}$ are simulated with the lowest variator size. The

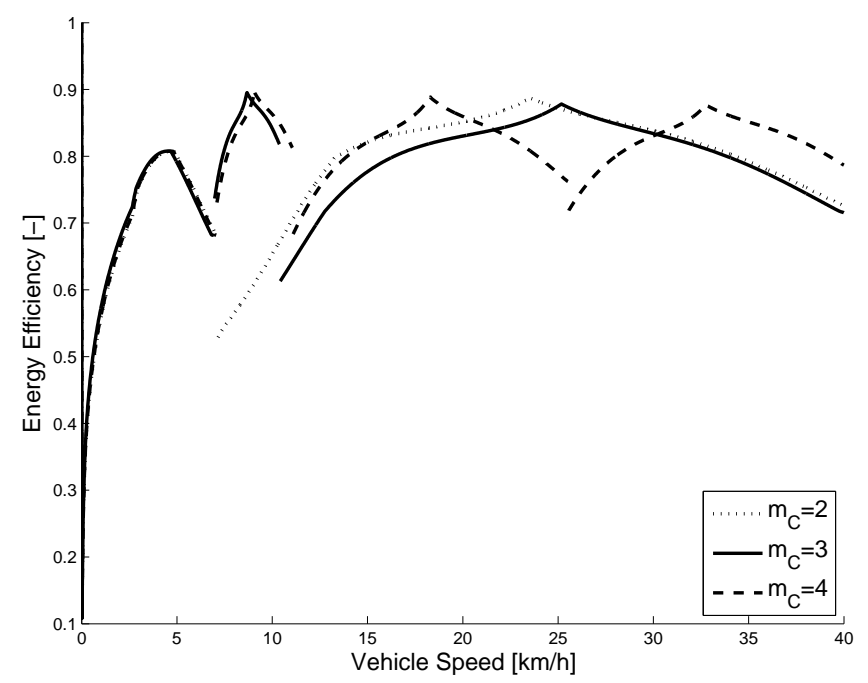

Figure 7: Simulation of an acceleration from 0 to maximum speed during maximum load conditions for wheel loader $C$. The peak efficiencies represent the full mechanical points where all power flows through the mechanical branch.

figure shows how the positioning of mode shifts is handled by the optimisation algorithm. The constraint for the bucket fill operation (eq. (4)) is often a limiting factor and most designs have the first mode shift close to $5 \mathrm{~km} / \mathrm{h}$. The other mode shifts are more cleverly positioned to match the full mechanical points at the same speeds as the peaks in fig. 4 . For the depicted case, $m=3$ has even better capabilities to match the typical operating behaviour than $m=4$, which is a remarkable result.

To identify the decreased individual energy efficiency of the applications, the optimised designs are simulated with two different variator sizes. Table 3 compares the equivalent energy consumption for the wheel loaders using a larger variator than actually needed to achieve the specified performance.

Table 3: Percentage of increase in energy consumption when using the largest variator instead of the smallest one.

\begin{tabular}{c|ccc}
\hline Wheel loader & A & B & C \\
\hline$m=2$ & $2.6 \%$ & $1.9 \%$ & $2.5 \%$ \\
\hline$m=3$ & $-1.4 \%$ & $2.1 \%$ & $3.3 \%$ \\
\hline$m=4$ & $-1.1 \%$ & $1.1 \%$ & $2.9 \%$ \\
\hline$m=5$ & $-1.0 \%$ & $1.5 \%$ & $3.0 \%$ \\
\hline$m=6$ & $-2.3 \%$ & $0.5 \%$ & $2.6 \%$ \\
\hline
\end{tabular}

Normally, a transmission with a smaller variator suffers from lower power losses, as the parasitic losses from the hydrostatic transmission are reduced. This seems to be the case for wheel loaders B and C. A small variator used in a large application, however, is forced to operate with smaller relative displacements which lowers the efficiency of the transmission. This is the reason why wheel loader A has a lower energy consumption when a larger variator is chosen. A special case is $m=2$ for wheel loader A, which suffers from poor energy efficiency in both cases. Table 4 displays the above discussed phenomenon for wheel loader A.

Table 4: Optimal $\varepsilon_{2, \text { min }}$ for wheel loader A.

\begin{tabular}{c|ccc}
\hline$\left[\boldsymbol{D}_{\boldsymbol{1}}, \boldsymbol{D}_{\mathbf{2}}\right]$ & {$[\mathbf{1 4 5}, \mathbf{1 7 0}]$} & {$[\mathbf{1 7 5}, \mathbf{2 1 5}]$} & {$[\mathbf{2 1 0}, \mathbf{2 8 0}]$} \\
\hline$m=2$ & 0.45 & 0.44 & 0.61 \\
\hline$m=3$ & 0.76 & 0.47 & 0.96 \\
\hline$m=4$ & 0.75 & 0.84 & 0.95 \\
\hline$m=5$ & 0.74 & 0.83 & 0.96 \\
\hline$m=6$ & 0.58 & 0.83 & 0.94 \\
\hline
\end{tabular}

\section{Conclusions}

In this paper, a design methodology for modular multiplemode hydromechanical transmissions has been proposed and implemented on the design of the "Jarchow-concept" for a range of wheel loader applications. The problem has been formulated into a multi-objective optimisation problem where energy efficiency and manufacturing costs of the product family are antithetic objectives. The implementation indicates that an optimised design of the modular transmission is difficult to achieve manually. Moreover, the number of degrees of freedom for the design increases when using a collective hydraulic variator, which further complicates the design task. This increases the need for an automated design process for the industrial designer. 


\section{Nomenclature}

\section{Quantities}

\begin{tabular}{lll}
\hline Designation & Denotation & Unit \\
\hline $\mathrm{C}$ & Cost & {$[-]$} \\
$\mathrm{D}$ & Hydraulic displacement & {$\left[\mathrm{cm}^{3} / \mathrm{rev}\right]$} \\
$\mathrm{F}$ & Force & {$[\mathrm{kN}]$} \\
$\mathrm{i}$ & Gear ratio & {$[-]$} \\
$\mathrm{m}$ & Number of modes & {$[-]$} \\
$\mathrm{P}$ & Power & {$[\mathrm{kW}]$} \\
$\mathrm{R}$ & Planetary gear ratio & {$[-]$} \\
$\mathrm{v}$ & Vehicle speed & {$[\mathrm{km} / \mathrm{h}]$} \\
$\mathrm{w}$ & Weight factor & {$[-]$} \\
$\varepsilon$ & Relative displacement & {$[-]$} \\
$\lambda$ & Weight factor & {$[-]$}
\end{tabular}

Indices

\begin{tabular}{ll}
\hline Designation & Denotation \\
\hline $\mathrm{g}$ & Global \\
$\mathrm{l}$ & Local
\end{tabular}

\section{References}

[1] Thomas Anderl, Jürgen Winkelhake, and Marcus Scherer. Power-split Transmissions For Construction Machinery. In 8th International Fluid Power Conference, pages 189-201, Dresden, Germany, 2012.

[2] D Mikeska and M Ivantysynova. Virtual Prototyping of Power Split Drives. In Workshop on Power Transmission and Motion Control, pages 95-111, Bath, UK, 2002.

[3] P Casoli, A Vacca, G L Berta, S Meleti, and M Vescovini. A Numerical Model for the Simulation of Diesel / CVT Power Split Transmission. In 8th International Conference on Engines for Automobiles, Capri, Naples, Italy, 2007.

[4] Torsten Kohmäscher. Modellbildung, Analyse und Auslegung Hydrostatischer Antriebsstrangkonzepte. $\mathrm{PhD}$ thesis, RWTH Aachen, Aachen, Germany, 2009.

[5] Alexander Krauss and Monika Ivantysynova. Power Split Transmissions Versus Hydrostatic Multiple Motor Concepts - A Comparative Analysis. SAE Transactions, 113(2), 2004.

[6] Blake Carl and Monika Ivantysynova. Comparison of Operational Characteristics in Power Split Continuously Variable Transmission. In Commercial Vehicle Engineering Congress and Exhibition, Chicago, Illinois, USA, 2006.

[7] S. Schembri Volpe, G. Carbone, M. Napolitano, and E. Sedoni. Design Optimization of Input and Output Coupled Power Split Infinitely Variable Transmissions. Journal of Mechanical Design, 131(11):111002, 2009.
[8] Alarico Macor and Antonio Rossetti. Optimization of Hydro-mechanical Power Split Transmissions. Mechanism and Machine Theory, 46(12):1901-1919, 2011.

[9] Antonio Rossetti and Alarico Macor. Multi-objective optimization of hydro-mechanical power split transmissions. Mechanism and Machine Theory, 62:112-128, April 2013.

[10] Karl Pettersson and Petter Krus. Design Optimization of Complex Hydromechanical Transmissions. ASME Journal of Mechanical Design, 2013. Accepted for publication.

[11] Alberto Jose and Michel Tollenaere. Modular and Platform Methods for Product Family Design : Literature Analysis. Journal of Intelligent Manufacturing, 16(3):371-390, 2005.

[12] K Steindorff. Methods for benchmarking the efficiency of mobile working machines and heavy duty vehicles. In 6th International Fluid Power Conference, pages 197207, Dresden, Germany, 2008.

[13] Bobbie Frank, Lennart Skogh, and Mats Alaküla. On Wheel Loader Fuel Efficiency Difference Due To Operator Behaviour Distribution. In 2nd International Commercial Vehicle Technology Symposium, CVT2012, Kaiserslauten, Germany, 2012.

[14] Reno Filla. Quantifying Operability of Working Machines. $\mathrm{PhD}$ thesis, Linköping University, Linköping, Sweden, 2011.

[15] Karl Pettersson and Petter Krus. Optimisation and Concept Sensitivity of Continuously Variable Hydromechanical Transmissions. In 8th International Conference on Fluid Power Transmission and Control, ICFP13, Hangzhou, China, 2013.

[16] Bosch Rexroth AG. Produktkatalog Mobilhydraulik. http://www.boschrexroth.com/mobile-hydraulics-catalog/, 2013.

[17] Petter Krus and Johan Andersson. Optimizing Optimization for Design Optimization. In ASME Design Engineering Technical Conferences and Computers and Information in Engineering Conference, Chicago, Illinois, USA, 2003. 